\title{
Necroptosis induced by RIPK3 requires MLKL but not Drp1
}

\author{
DM Moujalled ${ }^{1,2}$, WD Cook ${ }^{3}$, JM Murphy ${ }^{1,2}$ and DL Vaux ${ }^{*, 1,2}$
}

Necroptosis is a mechanism by which cells can kill themselves that does not require caspase activity or the presence of the pro-apoptotic Bcl-2 family members Bax or Bak. It has been reported that RIPK3 (receptor interacting protein kinase 3) activates MLKL (mixed lineage kinase domain-like) to cause cell death that requires dynamin-related protein 1 (Drp1), because survival was increased in cells depleted of Drp1 or treated with the Drp1 inhibitor mdivi-1. To analyze necroptosis in a system that does not require addition of tumor necrosis factor (TNF), we used a construct that allows RIPK3 to be induced in cells, and then dimerized via an $E$. coli gyrase domain fused to its carboxyl-terminus, using the dimeric gyrase binding antibiotic coumermycin. We have previously shown elsewhere that RIPK3 dimerized in this manner not only induces necroptosis but also apoptosis, which can be inhibited by the broad-spectrum caspase inhibitor Q-VD-OPh (QVD). In response to RIPK3 dimerization, wild-type mouse embryonic fibroblasts (MEFs) underwent cell death that was reduced but not completely blocked by QVD. In contrast, death upon dimerization of RIPK3 in MIkI ${ }^{-1-}$ MEFs was completely inhibited with QVD, confirming that MLKL is required for necroptosis. Similar to wild-type MEFs, most $\operatorname{Drp1}^{-/-}$MEFs died when RIPK3 was activated, even in the presence of QVD. Furthermore, overexpression of wild-type MLKL or dominant active mutants of MLKL (Q343A or S345E/S347E) caused death of wild-type and Drp1 ${ }^{-1-}$ MEFs that was not inhibited with QVD. These results indicate that necroptosis caused by RIPK3 requires MLKL but not Drp1.

Cell Death and Disease (2014) 5, e1086; doi:10.1038/cddis.2014.18; published online 27 February 2014

Subject Category: Experimental Medicine

Most cells in metazoans do not die because they are damaged or lack nutrients; they die because they kill themselves through activation of a mechanism that has evolved for that purpose. One of the best-characterized mechanisms for cell suicide involves release of proteins from the mitochondria via Bax or Bak, the two pro-apoptotic members of the Bcl-2 family. ${ }^{1,2}$ When released, cytochrome $c$ enters the cytoplasm and binds to Apaf-1, which activates caspase 9 and caspase 3 , and the cell dies by apoptosis. ${ }^{3,4}$ This cell death mechanism can be blocked by overexpression of $\mathrm{Bcl}-2$, or by deletion of genes for Bax and Bak.

Another way that apoptosis can be induced is following ligation of members of the tumor necrosis factor receptor (TNFR) superfamily known as 'death receptors' such as TNFR1, CD95, and TRAIL receptors that can signal FADD to activate caspase 8. $^{5-7}$ Yet another mechanism for cell death is via activation of caspase 1 by one of several kinds of inflammasomes, a process termed 'pyroptosis'. In these cases, cell death can be blocked by a broad-spectrum caspase inhibitor, such as zVAD-fmk or QVD-OPh. ${ }^{8-10}$

The term 'necroptosis' is used to describe a form of cell death that is not blocked by caspase inhibitors or by overexpression of anti-apoptotic Bcl-2 family members.
Typically, necroptosis has been observed when certain cell lines such as L929 fibroblasts or Jurkat T cells are exposed to TNF, CD95 Ligand, or TRAIL in the presence of a broadspectrum caspase inhibitor such as zVAD-fmk. ${ }^{11}$

Degterev et al. ${ }^{12}$ identified necrostatin-1 (nec-1) in a screen for small molecules that would inhibit death of monocytic U937 cells treated with TNF plus zVAD-fmk, and later showed that it functioned by inhibiting the kinase activity of receptor interacting protein kinase 1 (RIPK1). ${ }^{13}$ In a similar screen, Sun et al. ${ }^{14}$ studied necroptosis in colon cancer HT-29 cells and FADD mutant Jurkat cells treated with a combination of TNF, a smac-mimetic IAP antagonist, and zVAD-fmk, and found that RIPK3 (receptor interacting protein kinase 3) and MLKL (mixed lineage kinase domain-like) were required for cell death. In an accompanying paper, they reported that MLKL was activated by RIPK3, and killed by a process requiring PGAM5, dynamin-related protein 1 (Drp1), and reactive oxygen species. ${ }^{15}$

We previously reported that RIPK1 is not essential for TNF to trigger necroptosis of mouse embryonic fibroblasts (MEFs) when RIPK3 levels were elevated. ${ }^{16}$ Therefore, depending on the cellular context, necroptosis can be executed by a mechanism independent of RIPK1. In this study, we aimed

\footnotetext{
${ }^{1}$ Walter and Eliza Hall Institute of Medical Research, 1 G Royal Parade, Parkville, Melbourne, Victoria 3052, Australia; ${ }^{2}$ Department of Medical Biology, University of Melbourne, Parkville, Victoria 3050, Australia and ${ }^{3}$ La Trobe Institute for Molecular Science, La Trobe University, Kingsbury Drive, Bundoora, Victoria 3086, Australia

${ }^{*}$ Corresponding author: DL Vaux, Walter and Eliza Hall Institute of Medical Research, University of Melbourne, 1G Royal Parade, Parkville, Melbourne Victoria 3052, Australia. Tel: +61 39345 2941; Fax: +61 39347 0852; E-mail: vaux@ wehi.edu.au

Keywords: necroptosis; receptor interacting protein kinases; MLKL; caspases

Abbreviations: Drp1, dynamin-related protein 1; MLKL, mixed lineage kinase domain-like; RIPK3, receptor interacting protein kinase 3; RIPK1, receptor interacting protein kinase 1; TNF, tumor necrosis factor; TNFR1, tumor necrosis factor receptor 1

Received 18.12.13; accepted 08.1.14; Edited by G Melino
} 
to determine the requirements for MLKL and Drp1 for necroptosis.

In addition to triggering cell death, the receptors for TNF, CD95L, and TRAIL activate many other pathways, such as the $\mathrm{AP}-1$ (jun/fos) and NF- $\kappa \mathrm{B}$ transcription factor signaling pathways, ${ }^{17-19}$ that can cause a wide variety of changes in the cell. To avoid such epiphenomena, so that necroptosis can be induced without the need for addition of a death receptor ligand, we developed a model in which RIPK3 can be activated directly by induced dimerization. ${ }^{20}$ In this construct, a domain of $E$. coli gyrase is fused to the carboxyl terminus of RIPK3. When the divalent antibiotic coumermycin is added to cells, it binds to this fusion protein and causes it to dimerize. ${ }^{21}$

Sun et al. ${ }^{14}$ reported that for RIPK3 to cause cell death, it must phosphorylate MLKL, which associates with the mitochondrial phosphatase PGAM5, that in turn recruits Drp1 and dephosphorylates it at serine 637, thereby activating its GTPase activity. ${ }^{15}$ Active Drp1 was proposed to cause mitochondrial fragmentation that was essential for necroptosis to occur. ${ }^{15}$

To look at the requirements for MLKL and Drp1 in necroptosis induced by dimerization of RIPK3, we made stable cell lines from wild-type (WT), $\mathrm{MlkI}^{-/-}$, and $\mathrm{Drp1} 1^{- \text {I- }^{-}}$ MEFs that bore a 4-hydroxytamoxifen (4HT)-inducible RIPK3gyrase construct, and tested whether induction and forced dimerization of RIPK3 could induce cell death. We found that dimerization of RIPK3 induced death of WT, Drp1 ${ }^{-/-}$ and $\mathrm{MlkI}^{-/}$MEFs. In addition, overexpression of dominant active MLKL mutants (Q343A or S345E/S347E) was sufficient to induce death of both WT and Drp1 ${ }^{-/-}$MEFs. These results demonstrate that MLKL is required for RIPK3-induced necroptosis but Drp1 is not.

\section{Results}

Addition of TNF plus smac-mimetic causes WT, $\mathrm{MlkI}^{-1-}$, and $\operatorname{Drp1}^{-/-}$MEFs to die by apoptosis, rather than necroptosis. To confirm the genotype of $\mathrm{Mlkl}^{-/-}$and Drp1 $^{-/-}$MEFs, we analyzed them by western blot. Probing for MLKL or Drp1 confirmed the deletion of these genes in the respective gene-deleted MEFs (Figure 1a). However, we found that unlike WT MEFs, Drp1 ${ }^{-/-}$MEFs did not express detectable levels of RIPK3 or MLKL.

As we had previously found that treatment of MEFs with TNF plus smac-mimetic induced cell death by a caspase-8dependent apoptotic mechanism, ${ }^{16}$ we compared the sensitivity of WT, MlkI ${ }^{-1-}$, and Drp1 ${ }^{-1-}$ MEFs with killing by TNF plus smac-mimetic. We found that like WT MEFs, the MlkI ${ }^{-/-}$ MEFs died in response to TNF plus smac-mimetic (Figures $1 \mathrm{~b}$ and $\mathrm{c}$ ). Furthermore, because death of the cells could be inhibited by pretreatment with the broad-spectrum caspase inhibitor Q-VD-OPh, it was caspase dependent. In contrast to the WT and $M \mathrm{Kl}^{-1-} \mathrm{MEFs}$, at $24 \mathrm{~h}$, most of the Drp1 ${ }^{-/-}$ MEFs remained propidium iodide (PI) negative (viable) (Figure 1d). This is consistent with reports showing that apoptosis, measured by cytochrome $c$ release, caspase activation, and nuclear DNA fragmentation, is delayed in MEFs derived from $D r p 1^{-/-}$mice, but that cell death still occurred. ${ }^{22,23}$ We therefore extended treatment of the cells with TNF plus smac-mimetic, and found that increasing numbers of $D r p 1^{-1-}$ MEFs died by 48 and $72 \mathrm{~h}$ (Figure 1d).

To confirm that this delay in caspase-dependent apoptosis was due to absence of Drp1, we reconstituted the Drp1 $1^{-/-}$ MEFs with a doxycycline-inducible Drp1 construct (Figure 1e) and, following induction of Drp1 with doxycycline, treated the cells with TNF plus smac-mimetic. When Drp1 was restored, TNF plus smac-mimetic treatment induced cell death at a similar rate as in WT MEFs (Figure 1f). This indicates that neither MLKL nor Drp1 is essential for apoptosis induced by TNF plus smac-mimetic treatment, and this cell death is a caspase-dependent process.

Necroptosis induced by RIPK3 dimerization occurs independently of Drp1. The requirement for particular proteins to act downstream of MLKL in necroptosis is controversial. Wang et al. ${ }^{15}$ reported that for TNF plus smac-mimetic plus z-VAD-fmk to cause necroptosis, Drp1 had to be recruited to the necrosome by PGAM5S and to be activated by dephosphorylation. Moreover, knockdown of PGAM5S or Drp1 prevented the cells from dying, suggesting that the activation of Drp1 by PGAM5S was essential for death to occur. However, in both WT MEFs and L929 fibroblastoid cells, shRNA-mediated knockdown of PGAM5 failed to protect them from necroptosis induced by TNF plus smac-mimetic plus QVD, ${ }^{24}$ arguing that PGAM5 is dispensable for necroptosis.

To test the requirement of Drp1 in necroptosis in a system in which RIPK3 could be activated directly, and without the need for addition of TNF or smac-mimetic, we stably expressed a 4HT-inducible FLAG-tagged RIPK3-gyrase construct that could be induced to dimerize by addition of the antibiotic coumermycin. ${ }^{21}$ Elsewhere, we have reported that expression and dimerization of RIPK3-gyrase caused WT MEFs to die. ${ }^{20}$ Using this system, we introduced the RIPK3-gyrase construct into WT, $\mathrm{Mlkl}^{-1-}$, and Drp1 ${ }^{-/-}$MEFs, and performed western blot analysis to confirm inducible expression upon addition of $4 \mathrm{HT}$ (Figure 2a). We then took WT, MlkI ${ }^{-/-}$, and Drp1 ${ }^{-/-}$MEFs that had, or had not, been pretreated with QVD, and added $4 \mathrm{HT}$ and coumermycin to respectively induce and dimerize RIPK3.

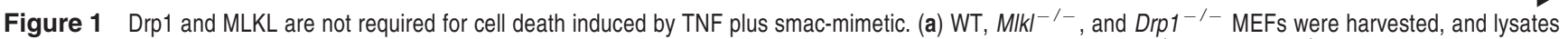
resolved on replicate gels were probed for MLKL, Drp1, or RIPK3. $\beta$-Actin was used as a loading control. (b-d) WT, MIkI ${ }^{-1-}$, and Drp $1^{-1-}$ MEFs were treated with $100 \mathrm{ng} / \mathrm{ml}$ TNF and/or $500 \mathrm{nM}$ smac-mimetic for 24,48 , or $72 \mathrm{~h}$. Cells were then stained with propidium iodide (PI) and analyzed by flow cytometry to detect loss of plasma membrane integrity. Error bars are S.E.M., where $n=3$ independently performed experiments (repeat $1=$ square, repeat $2=$ triangle, repeat $3=$ circle). (e) $D r p 1^{-1-}$ MEFs were infected with a lentiviral vector expressing FLAG-Drp1. Cells were induced with $1 \mu \mathrm{g} / \mathrm{ml}$ doxycycline for $24 \mathrm{~h}$, harvested, and lysates run on replicate gels were probed for FLAG or $\beta$-actin as a loading control. (f) Drp1 $1^{-/-}$MEFs were treated with $1 \mu \mathrm{g} / \mathrm{ml}$ doxycycline and/or $100 \mathrm{ng} / \mathrm{ml}$ TNF and $500 \mathrm{nM}$ smac-mimetic for $24 \mathrm{~h}$. Cells were stained with PI and analyzed by flow cytometry. Error bars are S.E.M., where $n=3$ independently performed experiments (repeat $1=$ square, repeat $2=$ triangle, repeat $3=$ circle)
} 
a
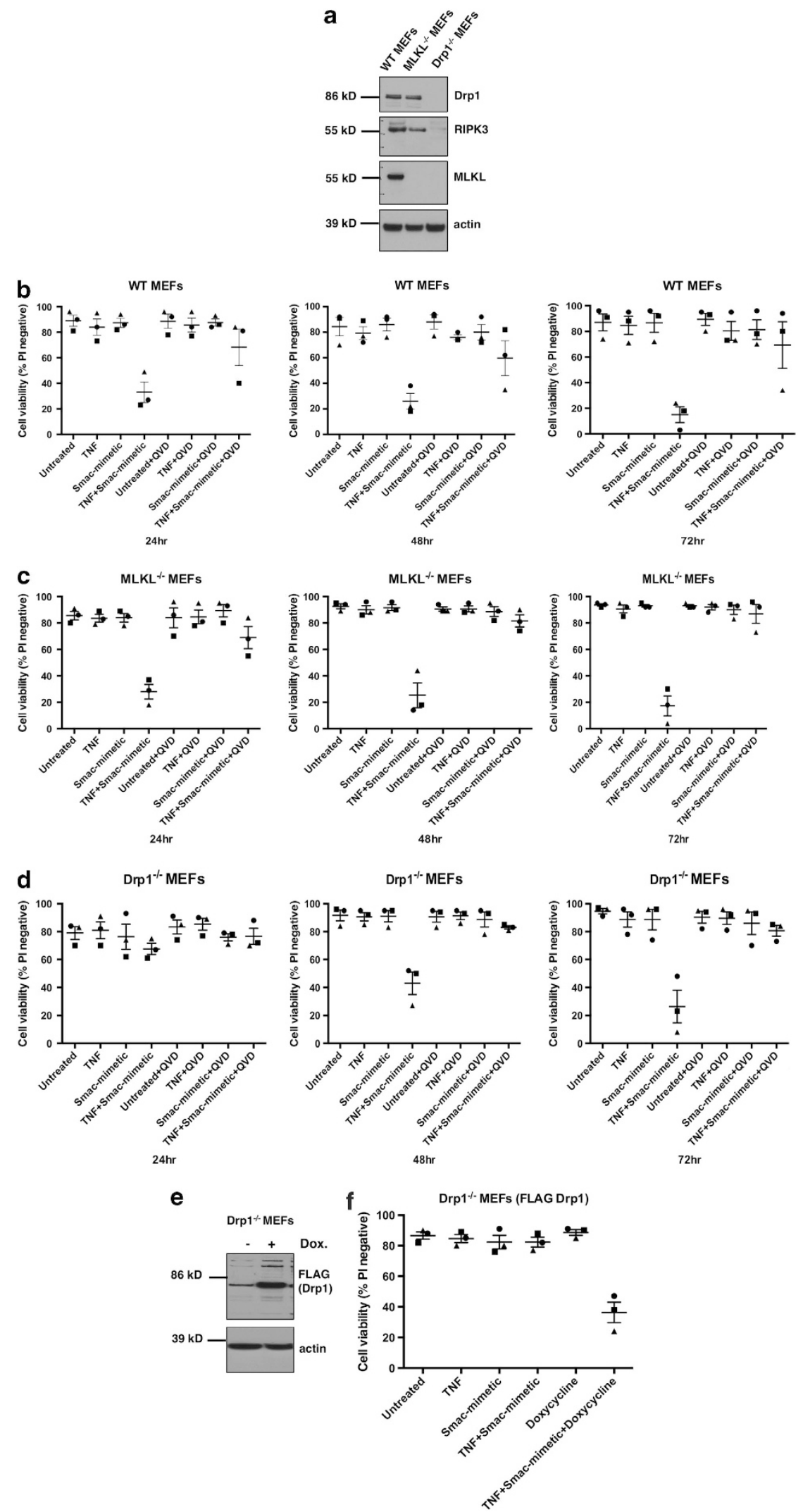

Drp ${ }^{-1-}$ MEFs

Drp $1^{-1-}$ MEFs 
Induction of RIPK3-gyrase alone caused little cell death among WT, MlkI ${ }^{-/-}$, and Drp1 ${ }^{-/-}$MEFs, whereas induction and dimerization of RIPK3 induced death of $\sim 70 \%$ of WT, $\mathrm{MlkI}^{-/-}$, and Drp1 $1^{-1-}$ MEFs by $24 \mathrm{~h}$ (Figure 2b). Because the percentage of WT MEFs that died was reduced by approximately half by pretreatment with QVD, we believe RIPK3 activation can induce both caspase-dependent and caspase-independent death mechanisms. When RIPK3gyrase was induced and dimerized in $\mathrm{Mlkl}^{-/-} \mathrm{MEFs}$, pretreatment with QVD gave complete protection, as the percentage of $\mathrm{PI}$ staining cells was no different to the untreated cells (Figure $2 b$, middle panel). This confirms that $M L K L$ is required for the caspase-independent cell death mechanism induced by RIPK3, that is, necroptosis, but not for the cells to die by a caspase-dependent mechanism, that is, apoptosis. These results are also consistent with our previous findings that RIPK3 can also activate caspase 8 to induce cell death by apoptosis. ${ }^{16,20}$
In contrast to the $\mathrm{MlkI}^{-/-} \mathrm{MEFs}$, some of the $\mathrm{Drp1} 1^{-/-}$ MEFs still died even when they were pretreated with QVD, suggesting that Drp1 is not essential for necroptosis.

We then tested whether inhibiting the GTPase domain of Drp1 could protect from RIPK3 dimerization-induced cell death. Inhibition of Drp1 by mdivi-1 was not able to increase cell survival (Figure 2c) or increase clonogenicity (Figure 2d). This indicates that inhibiting the GTPase domain of Drp1 does not block cell death induced by RIPK3 dimerization. As we had found that cell death is sometimes delayed but not abrogated in the $D r p 1^{-/-}$MEFs, we tested them further by inducing necroptosis directly by overexpression of MLKL.

Overexpression of a dominant active form of MLKL induces death of both WT and Drp1 ${ }^{-/-}$MEFs; Drp1 is not required for necroptosis. To examine whether MLKL can induce death of WT and Drp1 $1^{-1-}$ MEFs, we generated doxycycline-inducible constructs encoding WT MLKL,

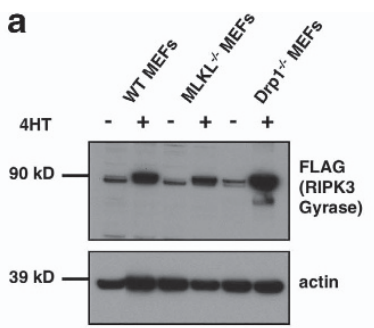

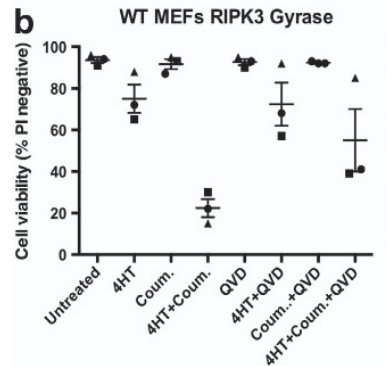

C

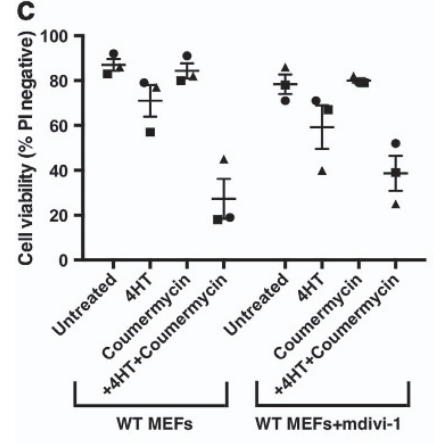

MLKL \% MEFs RIPK3 Gyrase
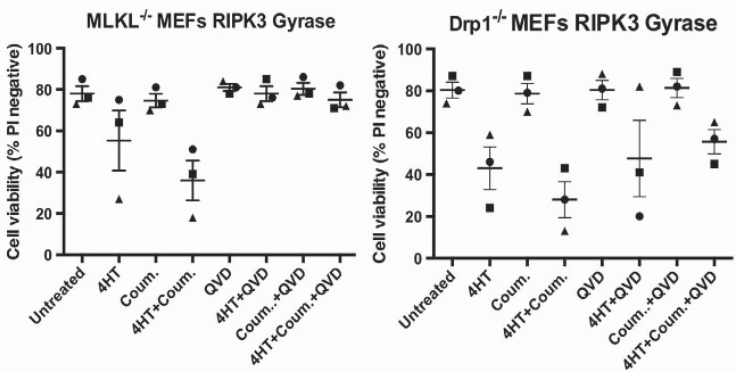

d

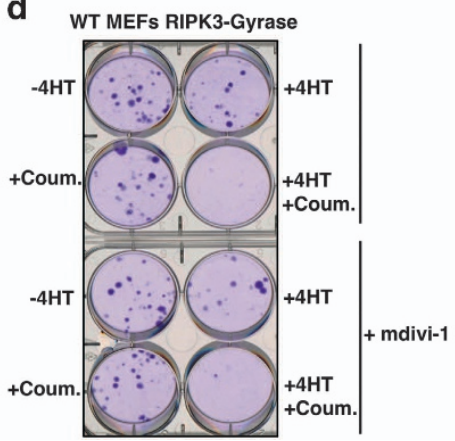

Figure 2 Necroptosis induced by RIPK3 dimerization occurs independently of Drp1. (a) WT, MIkl ${ }^{-/-}$, and Drp1 ${ }^{-/-}$MEFs were infected with a lentiviral vector expressing FLAG-tagged RIPK3-gyrase. Cells were induced with $10 \mathrm{nM} 4 \mathrm{HT}$ for $24 \mathrm{~h}$, harvested, and lysates on replicate gels were probed for FLAG or $\beta$-actin as a loading control. (b) Where indicated, cells were pretreated with $10 \mu \mathrm{M}$ QVD for $1 \mathrm{~h}$ and subsequently treated with $10 \mathrm{nM} 4 \mathrm{HT}$ and/or $700 \mathrm{nM}$ coumermycin for $24 \mathrm{~h}$. Cells were stained with PI and cell viability determined by flow cytometry. Error bars are S.E.M., where $n=3$ independently performed experiments (repeat $1=$ square, repeat $2=$ triangle, repeat $3=$ circle). (c) Inhibition of the GTPase domain of Drp1 does not protect from RIPK3 dimerization-induced cell death. WT MEFs expressing 4HT-inducible RIPK3gyrase were pretreated with $100 \mu \mathrm{M}$ of the Drp1 inhibitor mdivi-1 for $1 \mathrm{~h}$, followed by treatment with $10 \mathrm{nM} 4 \mathrm{HT}$ and $700 \mathrm{nM}$ coumermycin for $24 \mathrm{~h}$. Cells were stained with PI and analyzed by flow cytometry. Error bars are S.E.M., where $n=3$ independently performed experiments (repeat $1=$ square, repeat $2=$ triangle, repeat $3=$ circle). (d) WT MEFs expressing 4HT-inducible RIPK3-gyrase were pretreated with $100 \mu \mathrm{M}$ mdivi-1 for $1 \mathrm{~h}$, followed by treatment with $10 \mathrm{nM} 4 \mathrm{HT}$ and $700 \mathrm{nM}$ coumermycin for $24 \mathrm{~h}$. Cells were resuspended using trypsin, replated, and after 5 days stained with crystal violet to assess clonogenicity. Inhibition of Drp1 by mdivi- 1 was not able to protect clone-forming cells from death 
and dominant active (Q343A or S345E/S347E ('SSEE')) mutant forms of MLKL, and transfected them into WT and $\mathrm{Drp1}^{-/-}$MEFs. The dominant activity of Q343A MLKL has been described previously, ${ }^{24}$ whereas the substitution of serines for negatively charged Glu residues in the SSEE mutant was designed to activate MLKL by mimicking phosphorylation of S345 and S347, both of which are known to be RIPK3 substrates. ${ }^{24}$ Expression of these constructs upon addition of doxycycline was confirmed by western blotting (Figures $3 a$ and e). We then pretreated half of the cultures with QVD, and induced expression of WT or Q343A dominant active MLKL in the WT and Drp1 $1^{-/-}$MEFs. Induction of WT MLKL caused the WT MEFs to die, but there was more cell death following induction of the dominant active Q343A point mutant form of MLKL (Figure 3b). The mechanism of cell death was caspase independent (Figure $3 b$ ) because it was not inhibited by the same concentrations of QVD that completely protected $\mathrm{MlkI}^{-1-}$ MEFs from caspase 8-dependent apoptosis (Figure $2 \mathrm{~b}$ ).

Compared with the WT MEFs, fewer Drp1-/- MEFs were killed when WT or Q343A dominant active MLKL was induced (Figures $3 c$ and d, Dox stimulation). As we had found that the Drp1 $^{-/-}$MEFs did not express detectable levels of endogenous RIPK3, whereas the WT MEFs did (Figure 1a), we tested whether coexpression of FLAG-RIPK3-gyrase together with MLKL could increase the number of Drp $1^{-/-}$MEFs that died. As shown in Figures $3 c$ and d, induction of FLAG-RIPK3gyrase together with WT MLKL, or the dominant active Q343A MLKL, caused yet more death among the Drp1-/- MEFs and, because this was not inhibited by pretreatment with QVD, this indicates that they were dying by necroptosis, in the absence of Drp1. We then tested WT and Drp1 ${ }^{-1-}$ MEFs

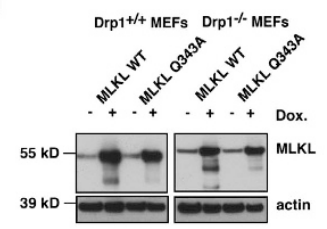

b

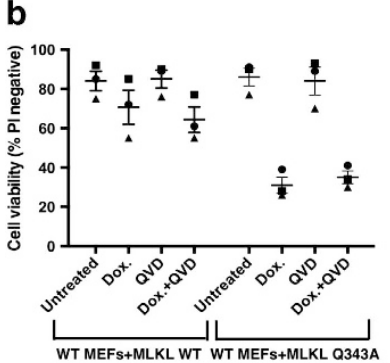

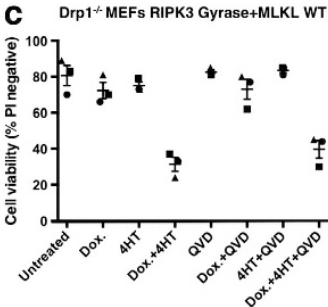

d

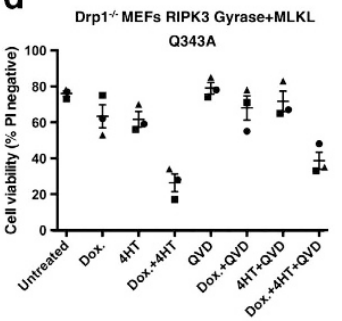

e

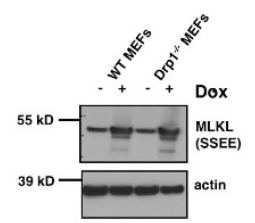

f

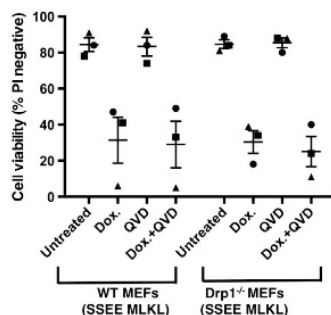

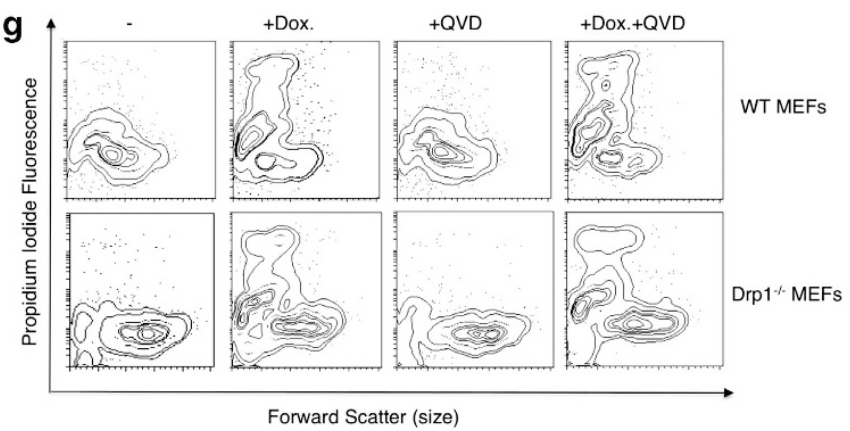

Figure 3 Overexpression of MLKL induces cell death of WT and Drp1 ${ }^{-/-}$MEFs. (a) WT and Drp $1^{-/-}$MEFs were infected with a lentiviral vector expressing WT MLKL or a dominant active MLKL (Q343A). Cells were treated with $1 \mu \mathrm{g} / \mathrm{ml}$ doxycycline for $24 \mathrm{~h}$ to induce MLKL expression. Lysates were harvested, run on replicate gels, and probed for MLKL or $\beta$-actin as a loading control. (b) WT MEFs, where indicated, were pretreated with $10 \mu \mathrm{M} \mathrm{QVD}$ for $1 \mathrm{~h}$ and subsequently treated with $1 \mu \mathrm{g} / \mathrm{ml}$ doxycycline for $24 \mathrm{~h}$. Cells were stained with propidium iodide (PI) and analyzed by flow cytometry. Error bars are S.E.M, where $n=3$ independently performed experiments (repeat $1=$ square, repeat $2=$ triangle, repeat $3=$ circle). (c and d) Where indicated, Drp $1^{-1}$ MEFs were pretreated with $10 \mu \mathrm{M} \mathrm{QVD} \mathrm{for} 1 \mathrm{~h}$ and subsequently treated with $1 \mu \mathrm{g} / \mathrm{ml}$ doxycycline (to induce MLKL expression) and/or $10 \mathrm{nM} 4 \mathrm{HT}$ (to induce RIPK3-gyrase expression) for $24 \mathrm{~h}$. Cells were stained with PI and analyzed by flow cytometry. Error bars are S.E.M., where $n=3$ independently performed experiments (repeat $1=$ square, repeat $2=$ triangle, repeat $3=$ circle). (e) WT and Drp $1^{-1-}$ MEFs were infected with a lentiviral vector bearing dominant active (SSEE) mutant MLKL. Cells were treated with $1 \mu \mathrm{g} / \mathrm{ml}$ doxycycline to induce SSEE MLKL expression. Lysates were harvested, run on replicate gels, and probed for MLKL or $\beta$-actin as a loading control. (f) Where indicated, WT and Drp $1^{-1-}$ MEFs were pretreated with $10 \mu \mathrm{M}$ QVD for $1 \mathrm{~h}$ and subsequently treated with $1 \mu \mathrm{g} / \mathrm{ml}$ doxycycline for $24 \mathrm{~h}$. Cells were stained with PI and analyzed by flow cytometry. Error bars are S.E.M., where $n=3$ independently performed experiments (repeat 1 = square, repeat $2=$ triangle, repeat $3=$ circle). $(\mathbf{g})$ Representative contour plots of cell death induced by MLKL (SSEE) in WT and Drp1 ${ }^{-1}-$ MEFs. Where indicated, WT and Drp1 $1^{-/-}$MEFs were pretreated with $10 \mu \mathrm{M} \mathrm{QVD}$ for $1 \mathrm{~h}$ and subsequently treated with $1 \mu \mathrm{g} / \mathrm{ml}$ doxycycline for $24 \mathrm{~h}$. Cells were stained with $\mathrm{PI}$ and analyzed by flow cytometry. Note the increase in PI-staining cells when MLKL (SSEE) is induced in both WT and Drp1 ${ }^{-/-}$MEFs, and this is not reduced by pretreatment with QVD 
stably expressing a MLKL SSEE mutant (Figure 3e). As shown in Figures $3 f$ and $g$, induction of SSEE MLKL in both WT and Drp1-/- MEFs caused cell death that was not inhibited by QVD, indicating that the cells were dying by necroptosis.

\section{Discussion}

Programmed necrosis or 'necroptosis' is a mechanism by which cells can kill themselves that does not require Bax or Bak, and does not depend on caspase activity. ${ }^{25}$ Instead, it has been proposed that necroptosis requires RIPK1, RIPK3, MLKL, PGAM5, and Drp1. ${ }^{14,15}$

Previously, we have shown that if levels of RIPK3 are elevated, TNF can trigger necroptosis in the absence of RIPK1, and hence the requirement for RIPK1 in necroptosis might not be absolute. There is, however, growing evidence that RIPK3 and MLKL are obligatory for necroptosis to occur. ${ }^{14,24}$ In contrast, one study examined the requirement for PGAM5 by knocking down its expression, and found that necroptosis was unaffected. ${ }^{24}$

Drp1 has been reported to be required for cell death in multiple circumstances. For example, Drp1 was reported to be essential for apoptosis induced by staurosporine, etoposide, and Fas ligation. ${ }^{26}$ However, these findings have been challenged by others who suggest that Drp1 is not required for Bax/Bak-dependent apoptosis. ${ }^{27}$

A requirement for Drp1 in caspase-independent cell death was reported by Bras et al. ${ }^{28}$ They reported that stimulation of chronic lymphocytic leukemia cells with an antibody to CD47 caused activation of serine proteases that allowed Drp1 to translocate to the mitochondria, resulting in their fragmentation, and consequently cell death. Knockdown of Drp1 expression, on the other hand, was shown by Bras et al. ${ }^{28}$ to protect these cells from cell death. Consistent with this report, Wang et al. ${ }^{15}$ found that in RIPK3-induced necrosis, PGAM5 dephosphorylated and hence activated Drp1, which was an obligatory step for necrosis execution.

Here, we directly tested the requirement for Drp1 in cell death induced by activation of RIPK3 by using Drp $1^{-1-} \mathrm{MEFs}^{22}$ bearing a $4 \mathrm{HT}$-inducible vector encoding a RIPK3-gyrase fusion protein that dimerizes in response to coumermycin.

Consistent with earlier reports showing that loss of Drp1 delays, but does not inhibit, caspase-dependent apoptosis, ${ }^{29,30}$ we found that the $\operatorname{Drp} 1^{-/}$MEFs died more slowly than WT MEFs when treated with TNF plus smac-mimetic, a treatment that causes caspase 8-dependent cell death. Whereas most of the WT MEFs died after $24 \mathrm{~h}$ of treatment, the Drp1 $1^{-/-}$MEFs began to die at $48 \mathrm{~h}$, with most dead by $72 \mathrm{~h}$. This shows that there is no absolute requirement for Drp1 for caspase 8-dependent apoptosis.

As we have reported elsewhere that dimerization of RIPK3 was sufficient to cause the death of WT MEFs, ${ }^{20}$ we tested whether RIPK3 dimerization could induce cell death of Drp1 ${ }^{-/-}$MEFs. Induction and dimerization of RIPK3 caused death of WT, $\mathrm{MlkI}^{-/-}$, and Drp1 ${ }^{-/-}$MEFs. Pretreatment with the caspase inhibitor QVD completely protected the $\mathrm{MlkI}^{-/-}$ MEFs, indicating that RIPK3 activation can activate caspase-dependent apoptosis, consistent with our previous observations. ${ }^{16,20}$ Furthermore, because QVD only gave partial protection to the WT and Drp1 $1^{-/-}$MEFs, RIPK3 activation must have also been able to trigger a caspaseindependent cell death mechanism that depended on the presence of MLKL.

Addition of QVD gave partial protection in both WT and Drp 1 ${ }^{-/-}$MEFs, whereas the MIkI ${ }^{-/-}$MEFs were completely protected. This reinforces the notion that MLKL is essential for necroptosis and is activated by phosphorylation by RIPK3, ${ }^{14,24}$ but shows that Drp1 is dispensable. This conclusion is also consistent with the failure of Mdivi-1, the specific Drp1 GTPase inhibitor, ${ }^{31,32}$ to prevent cell death, as determined by cell viability and clonogenicity assays.

We then tested whether increasing the level of MLKL was sufficient to cause the death of both WT and Drp1 ${ }^{-/-}$MEFs. Induction of WT MLKL was sufficient to cause death of some WT MEFs, with the dominant active MLKL (Q343A) causing more. Simultaneous induction of both RIPK3-gyrase and WT or Q343A MLKL caused yet more death of the Drp1-1MEFs, and because the cells were not protected by QVD, their death was also not dependent on caspase activity. Furthermore, following induction of MLKL SSEE, a more potent dominant active form of MLKL, we observed potent killing of WT and Drp1-1- MEFs even without overexpression of RIPK3, and this was also not blocked by QVD. These results provide compelling evidence that Drp1 is dispensable for necroptosis execution downstream of MLKL.

Our results question whether dephosphorylation of Drp 1 by $\mathrm{PGAM}^{15}$ and activation of Drp1 to induce mitochondrial fragmentation $^{28,33}$ are obligatory for caspase-independent cell death, but are consistent with RIPK3 and MLKL having essential roles.

\section{Materials and Methods}

Genetically modified cell lines and mice. Production of MEF lines has been described previously in detail. ${ }^{34}$ Primary MEFs were generated from E15.5 embryos and then infected with SV40 large T-antigen-expressing lentivirus to generate immortal cell lines. Mlkl ${ }^{-1-}$ MEFs were produced as previously described..$^{16}$ Drp $1^{-1-}$ MEFs were provided by Hiromi Sesaki (Oregon University, Eugene, OR, USA) and were produced as previously described. ${ }^{22}$

Cell Culture, transfections, constructs, and lentiviral infections. Cell lines were maintained at $37^{\circ} \mathrm{C}, 10 \% \mathrm{CO}_{2}$ in DMEM supplemented with $10 \%$ (v/v) fetal bovine serum (Gibco, Melbourne, VIC, Australia), $50 \mu \mathrm{g} / \mathrm{ml}$ penicillin G, $50 \mathrm{U} / \mathrm{ml}$ streptomycin, and $2 \mathrm{mM}$ L-glutamine. Mouse FLAG-RIPK3-gyrase was cloned into the $4 \mathrm{HT}$-inducible lentiviral vector $\mathrm{pF} 5 \times \mathrm{UAS}^{34,35}$ The cDNA encoding mouse Drp1 was obtained from David Chan via Addgene (Cambridge, MA, USA) (plasmid 34706) and mouse FLAG Drp1 was cloned into the doxycycline-inducible vector pF TRE3G PGK puro, ${ }^{16,24}$ a derivative of the $\mathrm{pF}$ $5 \times$ UAS puro vector ${ }^{34}$ in which the tetracycline-responsive element replaces $5 \times$ UAS, and the $3 G$ transactivator (Tet3G) $2 A$ peptide puro-resistance cassette expressed under the control of a PGK promoter replaces the SV40 Puro cassette. The cDNA-encoding mouse MLKL (residues 1-464; UniProt sequence Q9D2Y4-2; synthesized by DNA2.0, Menlo Park, CA, USA), and Q343A or SSEE mutants generated by oligonucleotide-directed PCR mutagenesis were cloned into pF TRE3G PGK puro and insert sequences were verified (Micromon DNA Sequencing Facility, Clayton, VIC, Australia).

Lentiviruses were generated by transfecting subconfluent $10 \mathrm{~cm}$ plates of 293T cells with vector plasmids together with the packaging constructs $p C M V-\triangle R 8$ and pVSV-G using Effectene (Qiagen, Chadstone, VIC, Australia) as described previously. ${ }^{35}$ After $48 \mathrm{~h}$, viral supernatants were collected, filtered, supplemented with $4 \mu \mathrm{g} / \mathrm{ml}$ polybrene, and added to the target MEFs. Stably infected cells were selected in the presence of $5 \mu \mathrm{g} / \mathrm{ml}$ puromycin and $300 \mu \mathrm{g} / \mathrm{ml}$ hygromycin B. Expression of pF $5 \times$ UAS-inducible constructs was induced with $10 \mathrm{nM} 4 \mathrm{HT}$ unless otherwise indicated. 
Antibodies and chemicals. Primary antibodies used for western blot analysis were anti-FLAG (F-3165; Sigma, Croydon, VIC, Australia), anti-Drp1 (611112; BD Biosciences, North Ryde, NSW, Australia), anti- $\beta$-actin (A-1978; Sigma), anti-RIPK3 (551042; Pharmingen, North Ryde, NSW, Australia), and antiMLKL (3H1, generated in-house; available as MABC604; Millipore, Kilsyth, VIC, Australia). The $4 \mathrm{HT}$, doxycycline, coumermycin, and Mdivi-1 were purchased from Sigma and Q-VD-OPh (OPH001) from SM Biochemicals (Anaheim, CA, USA).

Cell death assays. Cells were seeded at $\sim 40 \%$ confluency onto 12-well tissue culture plates and were allowed to settle for $16-20 \mathrm{~h}$. Where indicated, $10 \mathrm{nM} 4 \mathrm{HT}$ and/or $700 \mathrm{nM}$ coumermycin or $10 \mu \mathrm{M} \mathrm{Q}$-VD-OPh were added to cells for $24 \mathrm{~h}$ and cell death measured by uptake of PI using a FACScalibur flow cytometer (BD Biosciences). Per sample, 10000 events were collected, and the percentage of live cells (\% PI-negative cells) quantified using WEASEL software (version 2.2.2, WEHI, Melbourne, VIC, Australia).

Clonogenic survival assay. WT MEFs were plated at equal densities on six-well plates, pretreated with or without $100 \mu \mathrm{M}$ mdivi-1 for $1 \mathrm{~h}$, and then treated with $10 \mathrm{nM} 4 \mathrm{HT}$ and/or $700 \mathrm{nM}$ coumermycin to induce or dimerize RIPK3-gyrase for $24 \mathrm{~h}$. After treatment, cells were treated with trypsin, resuspended, washed, and replated. Cells were then grown for 5 days and fixed with glutaraldehyde, and colonies stained with $0.1 \%(\mathrm{w} / \mathrm{v})$ crystal violet.

Western blotting. Lysates were prepared in DISC lysis buffer $(20 \mathrm{mM}$ Tris-

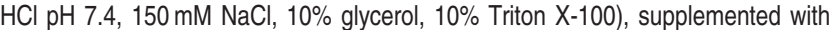
protease inhibitor cocktail (Roche, Dee Why, NSW, Australia). Protein samples were boiled in reducing loading dye before separation on $4-12 \%$ Bis-Tris polyacrylamide gels (Invitrogen, Mulgrave, VIC, Australia), and transferred to Hybond C nitrocellulose membrane (GE, Rydalmere, NSW, Australia) for incubation with specified antibodies. All membrane-blocking steps and antibody dilutions were performed using $5 \%(\mathrm{v} / \mathrm{v})$ skim milk in PBS containing $0.1 \%(\mathrm{v} / \mathrm{v})$ Tween-20 phosphate-buffered saline (PBST), and washing steps performed with PBST. Western blots were visualized by enhanced chemiluminescence (GE).

\section{Conflict of Interest}

The authors declare no conflict of interest.

Acknowledgements. This work was funded by NHMRC grants and fellowships 1016701 and 1020136, Cure Cancer Australia Foundation project grant 1050301, Cancer Council Victoria grant 1044722, and ARC fellowship FT100100100, and was made possible through Victorian State Government Operational Infrastructure Support and Australian Government NHMRC IRIISS We thank Hiromi Sesaki for providing the Drp $1^{-/}$MEFs and Toru Okamoto for the doxycycline-inducible vector.

1. Korsmeyer SJ, Wei MC, Saito M, Weiler S, Oh KJ, Schlesinger PH. Pro-apoptotic cascade activates $\mathrm{BID}$, which oligomerizes BAK or BAX into pores that result in the release of cytochrome c. Cell Death Differ 2000; 7: 1166-1173.

2. Zong WX, Lindsten $T$, Ross AJ, MacGregor GR, Thompson CB. BH3-only proteins that bind pro-survival $\mathrm{Bcl}-2$ family members fail to induce apoptosis in the absence of $\mathrm{Bax}$ and Bak. Genes Dev 2001; 15: 1481-1486.

3. Zou H, Li Y, Liu X, Wang X. An APAF-1.cytochrome c multimeric complex is a functional apoptosome that activates procaspase-9. J Biol Chem 1999; 274: 11549-11556.

4. Li P, Nijhawan D, Budihardjo I, Srinivasula SM, Ahmad M, Alnemri ES et al. Cytochrome C and dATP-dependent formation of Apaf-1/caspase-9 complex initiates an apoptotic protease cascade. Cell 1997; 91: 479-489.

5. Bodmer JL, Holler N, Reynard S, Vinciguerra P, Schneider P, Juo P et al. TRAIL receptor-2 signals apoptosis through FADD and caspase-8. Nat Cell biol 2000; 2: 241-243.

6. Ashkenazi A, Dixit VM. Death receptors: signaling and modulation. Science 1998; 281: 1305-1308

7. Juo P, Kuo CJ, Yuan J, Blenis J. Essential requirement for caspase-8/FLICE in the initiation of the Fas-induced apoptotic cascade. Curr Biol 1998; 8: 1001-1008.

8. Fernandes-Alnemri T, Wu J, Yu JW, Datta P, Miller B, Jankowski W et al. The pyroptosome: a supramolecular assembly of ASC dimers mediating inflammatory cell death via caspase-1 activation. Cell Death Differ 2007; 14: 1590-1604

9. Sokolovska A, Becker CE, Ip WK, Rathinam VA, Brudner M, Paquette N et al. Activation of caspase- 1 by the NLRP3 inflammasome regulates the NADPH oxidase NOX2 to control phagosome function. Nat Immunol 2013; 14: 543-553.
10. Vince JE, Wong WW, Gentle I, Lawlor KE, Allam R, O'Reilly L et al. Inhibitor of apoptosis proteins limit RIP3 kinase-dependent interleukin-1 activation. Immunity 2012; 36: 215-227.

11. Caserta TM, Smith AN, Gultice AD, Reedy MA, Brown TL. Q-VD-OPh a broad spectrum caspase inhibitor with potent antiapoptotic properties. Apoptosis 2003; 8: 345-352.

12. Degterev A, Huang Z, Boyce M, Li Y, Jagtap P, Mizushima N et al. Chemical inhibitor of nonapoptotic cell death with therapeutic potential for ischemic brain injury. Nat Chem Biol 2005; 1: 112-119.

13. Degterev A, Hitomi J, Germscheid M, Ch'en IL, Korkina O, Teng X et al. Identification of RIP1 kinase as a specific cellular target of necrostatins. Nat Chem Biol 2008; 4: 313-321.

14. Sun L, Wang H, Wang Z, He S, Chen S, Liao D et al. Mixed lineage kinase domain-like protein mediates necrosis signaling downstream of RIP3 kinase. Cell 2012; 148: 213-227.

15. Wang $Z$, Jiang $H$, Chen S, Du F, Wang X. The mitochondrial phosphatase PGAM5 functions at the convergence point of multiple necrotic death pathways. Cell 2012; 148: 228-243.

16. Moujalled DM, Cook WD, Okamoto T, Murphy J, Lawlor KE, Vince JE et al. TNF can activate RIPK3 and cause programmed necrosis in the absence of RIPK1. Cell Death Dis 2013: 4: e465

17. Liu ZG, Hsu H, Goeddel DV, Karin M. Dissection of TNF receptor 1 effector functions: JNK activation is not linked to apoptosis while NF-kappaB activation prevents cell death. Cell 1996; 87: 565-576.

18. Micheau O, Tschopp J. Induction of TNF receptor I-mediated apoptosis via two sequential signaling complexes. Cell 2003; 114: 181-190.

19. Varfolomeev EE, Ashkenazi A. Tumor necrosis factor: an apoptosis JuNKie? Cell 2004; 116: $491-497$

20. Cook WD, Moujalled DM, Ralph T, Lock P, Young SN, Murphy JM et al. RIPK1 and RIPK3-induced cell death mode is determined by substrate availability. Cell Death Diff (in revision).

21. Farrar MA, Alberol-lla J, Perlmutter RM. Activation of the Raf-1 kinase cascade by coumermycin-induced dimerization. Nature 1996; 383: 178-181.

22. Wakabayashi J, Zhang Z, Wakabayashi N, Tamura Y, Fukaya M, Kensler TW et al. The dynamin-related GTPase Drp1 is required for embryonic and brain development in mice. J Cell Biol 2009; 186: 805-816.

23. Ishihara N, Nomura M, Jofuku A, Kato H, Suzuki SO, Masuda K et al. Mitochondrial fission factor Drp1 is essential for embryonic development and synapse formation in mice. Nat Cell Biol 2009; 11: 958-966.

24. Murphy JM, Czabotar PE, Hildebrand JM, Lucet IS, Zhang JG, Alvarez-Diaz S et al. The pseudokinase MLKL mediates necroptosis via a molecular switch mechanism. Immunity 2013; 39: 443-453

25. Vanlangenakker N, Vanden Berghe T, Vandenabeele P. Many stimuli pull the necrotic trigger, an overview. Cell Death Diff 2012; 19: 75-86.

26. Frank S, Gaume B, Bergmann-Leitner ES, Leitner WW, Robert EG, Catez F et al. The role of dynamin-related protein 1, a mediator of mitochondrial fission, in apoptosis. Dev Cell 2001; 1: 515-525.

27. Parone PA, James DI, Da Cruz S, Mattenberger Y, Donze O, Barja F et al. Inhibiting the mitochondrial fission machinery does not prevent Bax/Bak-dependent apoptosis. Mol Cell Biol 2006; 26: 7397-7408

28. Bras M, Yuste VJ, Roue G, Barbier S, Sancho P, Virely C et al. Drp1 mediates caspaseindependent type III cell death in normal and leukemic cells. Mol Cell Biol 2007; 27: 7073-7088.

29. Estaquier J, Arnoult D. Inhibiting Drp1-mediated mitochondrial fission selectively prevents the release of cytochrome c during apoptosis. Cell Death Diff 2007; 14: 1086-1094.

30. Arnoult D, Rismanchi N, Grodet A, Roberts RG, Seeburg DP, Estaquier J et al. Bax/Bak-dependent release of DDP/TIMM8a promotes Drp1-mediated mitochondrial fission and mitoptosis during programmed cell death. Curr Biol 2005; 15: 2112-2118.

31. Cassidy-Stone A, Chipuk JE, Ingerman E, Song C, Yoo C, Kuwana T et al. Chemical inhibition of the mitochondrial division dynamin reveals its role in Bax/Bak-dependent mitochondrial outer membrane permeabilization. Dev Cell 2008; 14: 193-204.

32. Tanaka A, Youle RJ. A chemical inhibitor of DRP1 uncouples mitochondrial fission and apoptosis. Mol Cell 2008; 29: 409-410.

33. Barbier S, Chatre L, Bras M, Sancho P, Roue G, Virely C et al. Caspase-independent type III programmed cell death in chronic lymphocytic leukemia: the key role of the F-actin cytoskeleton. Haematologica 2009; 94: 507-517.

34. Vince JE, Wong WW, Khan N, Feltham R, Chau D, Ahmed AU et al. IAP antagonists target clAP1 to induce TNFalpha-dependent apoptosis. Cell 2007; 131: 682-693.

35. Callus BA, Ekert PG, Heraud JE, Jabbour AM, Kotevski A, Vince JE et al. Cytoplasmic p53 is not required for PUMA-induced apoptosis. Cell Death Diff 2008; 15: 213-215.

(c) (1) (2) Cell Death and Disease is an open-access journal published by Nature Publishing Group. This work is licensed under a Creative Commons Attribution-NonCommercialShareAlike 3.0 Unported License. To view a copy of this license, visit http://creativecommons.org/licenses/by-nc-sa/3.0/ 